\title{
Investigación sobre manejo forestal a largo plazo en Patagonia Sur - Argentina: Lecciones del pasado, desafíos del presente
}

\author{
Long-term forest management research in South Patagonia - Argentina: \\ Lessons from the past, challenges from the present
}

\author{
GUILLERMO J. MARTÍNEZ-PASTURR ${ }^{1,2, ~ *}$, MARÍA V. LENCINAS', 2, PABLO L. PERI ${ }^{2,3}$, \\ JUAN M. CELLINI ${ }^{4} \&$ ALICIA MORETTO ${ }^{1,2}$
}

\author{
${ }^{1}$ Centro Austral de Investigaciones Científicas (CADIC), Houssay 200 (9410) Ushuaia, Tierra del Fuego, Argentina \\ ${ }^{2}$ Consejo Nacional de Investigaciones Científicas y Técnicas (CONICET) \\ ${ }^{3}$ Instituto Nacional de Tecnología Agropecuaria (INTA) y Universidad Nacional de la Patagonia Austral (UNPA), \\ Río Gallegos, Santa Cruz, Argentina \\ ${ }^{4}$ Universidad Nacional de La Plata (UNLP), La Plata, Buenos Aires, Argentina \\ *Autor correspondiente: cadicforestal@cadic.gov.ar
}

\begin{abstract}
RESUMEN
Argentina ha basado su economía en la agricultura y el ganado, donde el bosque ha sido visto como un obstáculo para el desarrollo. La cosecha forestal en Patagonia Sur ha sido una actividad basada en la colonización y explotación de nuevas áreas. Esta se realiza principalmente en bosques primarios sin ningún manejo silvícola posterior. A pesar de que el manejo actual no es sustentable, la investigación científica ha definido las bases para conservar, proteger y mejorar las propuestas de manejo forestal para los bosques nativos de Patagonia Sur. Las primeras parcelas de estudio a largo plazo (1965-1966) basaron su monitoreo en parámetros económicos y estructura forestal, estableciéndose cerca de veinte parcelas en bosques fiscales de Tierra del Fuego. La mayoría fueron destruidas o discontinuadas debido a la falta de planificación del uso de la tierra, escaso interés social en la investigación, falta de compromiso de instituciones, y poca colaboración con empresas forestales. Posteriormente, un segundo grupo de nueve parcelas se estableció en Patagonia Sur (1993-2004) basado en la colaboración entre empresas privadas e instituciones nacionales. Los objetivos incluyeron parámetros económicos y ecológicos. Las parcelas se establecieron en tierras privadas conjuntamente con empresas privadas y estancieros, mediante la firma de acuerdos y proyectos de investigación. Los principales problemas encontrados fueron: falta de compromiso de las administraciones forestales, ausencia de proyectos financiados a largo plazo, y que la responsabilidad de las parcelas continúa bajo la iniciativa personal de los investigadores participantes. El estudio de las parcelas permitió: (i) proponer nuevos sistemas de regeneración basados en la maximización del rendimiento y el mejoramiento del valor de conservación de los bosques manejados (e.g., retención variable y sistemas silvopastoriles), (ii) determinar la factibilidad económica de aplicación de tratamientos intermedios, (iii) determinar líneas base y definir los impactos de distintos sistemas silvícolas, (iv) proponer metodologías de monitoreo de amplio espectro y establecer áreas demostrativas de manejo, y (v) proveer áreas y conocimientos para entrenar profesionales en prácticas de manejo forestal y conservación de la biodiversidad. Las parcelas permanentes ya establecidas en los bosques de Nothofagus de Patagonia Sur y las bases de datos obtenidas podrían servir como punto de partida para definir un núcleo regional que pueda participar en el International Long Term Ecological Research para asegurar el uso sustentable de los bosques nativos.
\end{abstract}

Palabras clave: biodiversidad, bosques de Nothofagus, compañías privadas, monitoreo, silvicultura.

\section{ABSTRACT}

Argentina has based its economy in agriculture and cattle production seeing forests as one of the main obstacles for future development. Forest harvesting in South Patagonia has been an activity based on colonization and exploitation of new areas. Harvesting is mainly carried out in old-growth forests without silviculture management after the first cuts. Beyond this mismanagement, scientific research defined the basis to conserve, protect and improve the present forest management for the South Patagonia native forests. The first long-term study permanent plots (1965-1966) monitored only economic and forest structure parameters, and near twenty plots were established in public forested lands of Tierra del Fuego. Most of these plots were destroyed or discontinued due to a lack of land use planning, scarce social interest for long- 
term researches, absence of commitment of institutions, and few contributions of forest companies. After this, a second group of nine long-term study permanent plots were established in South Patagonia (19932004) based in the collaboration between private companies and national research institutions. The objectives included economic as well as ecological parameters. The new plots were established on private lands based on several agreements and joint venture projects between national institutions, private companies and ranch owners. The main limitations in these processes were: a lack of commitment from the provincial forest administrations, absence of long-term financed projects and a clear forest policy from the government, and that continuity of plot measurements rely only under the responsibility of researchers. The study of the long-term plots allowed: (i) to propose new harvesting systems by maximizing yield and improving conservation value of managed forests (e.g., variable retention and silvopastoral systems), (ii) to determine the economical feasibility of the intermediate treatments, (iii) to define base-lines and impacts of different silvicultural treatments, (iv) to propose a wide spectrum of monitoring methodologies and establish demonstrative areas of forest management, and (v) to provide areas and knowledge to train professionals in forest management and biodiversity conservation practices. The surviving permanent plots in Nothofagus forest in South Patagonia and the obtained information could be used as a starting point to define a regional nucleus that could participate in the International Long Term Ecological Research to improve the use of native forests.

Key words: biodiversity, monitoring, Nothofagus forests, private companies, silviculture.

\section{COLONIZACIÓN Y EXPLOTACIÓN FORESTAL EN PATAGONIA SUR}

La Argentina históricamente basó su economía en la ganadería y la agricultura, viéndose al bosque como un obstáculo para el desarrollo económico. En Patagonia Sur, la remoción del bosque seguida de incendios y su transformación en pastizales eran aceptadas como prácticas comunes a principios del siglo pasado (Rothkugel 1916), pero posteriormente se puso en evidencia su incompatibilidad con el manejo forestal sustentable (Alfonso 1942, Cozzo et al. 1967, Alonso et al. 1968).

Los primeros aprovechamientos de madera en Patagonia Sur se llevaron a cabo en forma de talas rasas (Peri et al. 2002, Gea et al. 2004), y debido al sobrepastoreo posterior, dichas áreas fueron transformadas a la ganadería. Posteriormente, se propuso la aplicación de diferentes métodos silvícolas (Costantino 1950), pero estos siempre fueron aplicados bajo un concepto de obtener el máximo beneficio económico con el menor costo (Alfonso 1942). El aprovechamiento forestal aplicado en Patagonia Sur, fue y continúa siendo, un esquema basado en la colonización de nuevas áreas y basado en la explotación forestal sin un esquema de manejo sustentable (Martínez-Pastur \& Lencinas 2005). La cosecha se realiza principalmente sobre áreas de bosques primarios sin manejo previo, donde no se lleva a cabo ningún manejo silvícola posterior. Por otra parte, durante la última década del siglo pasado se propuso un cambio de concepción en el manejo del bosque (e.g.,
Franklin et al. 1997), donde las variables ecológicas y sociales adquirieron mayor importancia en el diseño de nuevas propuestas de manejo silvícola (Martínez-Pastur \& Lencinas 2005, Peri et al. 2005, Peri 2005, 2006, González et al. 2006).

\section{LA INVESTIGACIÓN FORESTAL EN ARGENTINA Y PATAGONIA SUR}

La investigación forestal en la Argentina se relacionó en un comienzo con la administración del recurso forestal. Sin embargo, los organismos administrativos fueron afectados por diversos cambios en el tiempo, variando sus funciones y objetivos de acuerdo a las políticas nacionales y regionales. Los primeros trabajos publicados (e.g., Alfonso 1942) fueron llevados a cabo por la "Sección Técnica de Bosques” creada en 1932 dentro de la "Dirección de Tierras - Ministerio de Agricultura de la Nación”, bajo la supervisión de investigadores extranjeros, muchos de ellos croatas. Posteriormente la "Dirección Forestal de la Nación” (1943) y la "Administración Nacional de Bosques” (1948 - Ley Nacional N ${ }^{\circ}$ 13,273) buscando el ordenamiento de los bosques fiscales bajo un criterio de sustentabilidad y abastecimiento de mercados (Piterbarg 1965), impulsaron el estudio de los bosques nativos en todo el país y en Patagonia en particular, vinculando para ello a Universidades Nacionales (e.g., Costantino 1950, Cozzo et al. 1967, 1969, Alonso et al. 1968). Esta vinculación continuó con los 
sucesivos organismos de gestión (e.g., Mutarelli \& Orfila 1971, 1973), a través del "Servicio Forestal Nacional" (1968), el "Servicio Nacional Forestal" (1969) y el "Instituto Forestal Nacional" (IFONA) (1973 Ley Nacional $\left.\mathrm{N}^{\circ} 20,531\right)$. El IFONA lideró la investigación forestal en Patagonia Sur hasta su disolución en 1991.

A partir de entonces, instituciones nacionales como el "Instituto Nacional de Tecnología Agropecuaria” (INTA), la "Secretaría de Recursos Naturales y Ambiente Humano” (1991 - Decreto Nacional-Ley N ${ }^{\circ}$ 2,419) transformada luego en la "Secretaría de Ambiente y Desarrollo Sustentable de la Nación" (SAyDS), el "Consejo Nacional de Investigaciones Científicas y Técnicas” (CONICET), la "Administración de Parques Nacionales" (APN), Universidades Nacionales, así como instituciones y centros de investigación, provinciales y regionales (e.g., "Centro de Investigación Forestal", "Corporación Forestal Neuquina" o el "Centro de Investigación y Extensión Forestal Andino Patagónico") continuaron con las investigaciones forestales en Patagonia. Entre las iniciativas encaradas para la Patagonia, se destaca un proyecto (Proyecto de Investigación Aplicada a los Recursos Forestales Nativos PIARFON) creado por la SAyDS, que estableció las bases para la investigación del manejo forestal a largo plazo en Patagonia a través de la firma de convenios específicos por un lapso de 15 años con instituciones nacionales y establecimientos agropecuarios.

\section{INSTALACIÓN DE PARCELAS PERMANENTES EN PATAGONIA SUR: LECCIONES DEL PASADO}

A pesar de la falta de un manejo sustentable que ha caracterizado al aprovechamiento forestal en Patagonia Sur, la investigación forestal sentó las bases científicas para conservar, proteger y mejorar el manejo de sus bosques nativos (Costantino 1950, Piterbarg 1965, Mutarelli \& Orfila 1969). Las primeras parcelas permanentes de estudio a largo plazo (Tabla 1) tuvieron como objetivos a parámetros económicos y de estructura forestal (e.g., volumen total y estructura de edades del rodal, cantidad y calidad de volumen aprovechable). El principal objetivo era transformar los bosques primarios en bosque manejados tendientes a la normalidad (bosques secundarios) (Cozzo et al. 1967, 1969). Para ello se eligieron parcelas que representaran el ciclo forestal a lo largo de un gradiente de edades de modo de obtener resultados en corto-mediano plazo (Mutarelli \& Orfila 1969). En Tierra del Fuego se establecieron cerca de 20 parcelas de ensayo en bosques fiscales con diferentes objetivos de estudio: (i) ensayo de diferentes métodos de regeneración (e.g., talas rasas y cortas de selección), (ii) aplicación de tratamientos intermedios (e.g., raleos), (iii) clausuras contra animales domésticos, e (iv) introducción de especies forestales exóticas (e.g., Pseudotsuga menziesii [Mirb.] Franco).

Estas primeras parcelas se instalaron en las cercanías de los bosques de Nothofagus pumilio (Poepp \& Endl.) Krasser y $N$. betuloides (Mirb.) Oersted aprovechados por los aserraderos de la época, durante la década del '60 (Tabla 1, Fig. 1). Una parte importante de los ensayos (parcelas de Laguna Negra, Lapataia, Ensenada, Lago Roca y Cañadón del Toro) se encuentran actualmente bajo la jurisdicción del Parque Nacional Tierra del Fuego (Ley Nacional $\mathrm{N}^{\circ}$ 15,554), lo que impidió futuras intervenciones silvícolas en esos rodales debido a su localización en áreas restringidas. Otros ensayos (parcelas de Monte Redondo, Río Tristen, Vega Café y Tierra Mayor) fueron parcialmente destruidos por la construcción de un gasoducto o quedaron incluidos dentro de una reserva natural y paisajística de uso exclusivamente turístico (1994 - Decreto Provincial No 2,256). Tres parcelas quedaron intactas, pero en solo una de ellas (parcela de Agua Blanca) se continuó los estudios (Cozzo et al. 1967, 1969, Mutarelli \& Orfila 1969). En Agua Blanca (16 ha) se realizaron talas rasas (1965), y posteriormente, se establecieron ensayos de seis sistemas de raleos sobre la regeneración establecida (1985) que incluían propuestas clásicas (distanciamientos fijos y selección) y no tradicionales (fajas con diferentes orientaciones y talas rasas en un diseño de dameros), mientras que un segundo raleo y poda fue realizado en 1999-2000 (MartínezPastur et al. 2001)

El fracaso en la implementación de estas primeras parcelas permanentes de estudio a 
largo plazo se debió a cuatro factores principales: (i) la falta de planificación en el uso de la tierra por parte del Gobierno Provincial de Tierra del Fuego; (ii) el escaso interés de la sociedad en la conservación del medio ambiente y la investigación forestal a largo plazo, (iii) la falta de compromiso de las principales instituciones científicas, así como de los administradores del recurso forestal, para financiar estudios forestales a largo plazo, y (iv) la escasa participación de dueños de campos y empresas forestales. Las diferentes instituciones responsables de la instalación de los ensayos (Tabla 1, Fig. 1) nunca establecieron programas para asegurar su mantenimiento y plan de monitoreo, quedando la continuidad de la medición de las parcelas bajo la exclusiva responsabilidad e iniciativa de los propios investigadores científicos.

\section{IMPORTANCIA DE LA COLABORACIÓN ENTRE EMPRESAS PRIVADAS E INSTITUCIONES DE INVESTIGACIÓN: DESAFÍOS DEL PRESENTE}

A partir de la disolución de IFONA, el INTA estableció un programa de investigación forestal en Patagonia que incluyó una estrecha

TABLA 1

Parcelas de estudio de manejo forestal a largo plazo en bosques de Nothofagus pumilio en Patagonia Sur.

Long-term forest management study plots in Nothofagus pumilio forests of Southern Patagonia.

\begin{tabular}{|c|c|c|c|c|}
\hline Parcela & Año & Especie & Objetivos de manejo & Provincia \\
\hline Laguna Negra ${ }^{1,3}$ & 1965 & N. pumilio y $N$. betuloides & Raleos & Tierra del Fuego \\
\hline Río Tristen - Vega Café1,3 & 1965 & N. pumilio & Talas rasas & Tierra del Fuego \\
\hline Lago Roca ${ }^{1,2,3}$ & 1965 & N. pumilio y $N$. betuloides & Cortas de regeneración & Tierra del Fuego \\
\hline Río Tierra Mayor ${ }^{1,3}$ & 1965 & N. pumilio & Talas rasas & Tierra del Fuego \\
\hline Agua Blanca $1,3,4$ & 1965 & N. pumilio & Talas rasas, raleos y podas & Tierra del Fuego \\
\hline Monte Redondo ${ }^{1,2,3}$ & 1966 & N. pumilio & Talas rasas & Tierra del Fuego \\
\hline Cañadón del Toro ${ }^{1,3}$ & 1966 & N. pumilio & Raleos y cortas de regeneración & Tierra del Fuego \\
\hline Lapataia - Ensenada ${ }^{1,2,3}$ & 1966 & N. pumilio y $N$. betuloides & Raleos & Tierra del Fuego \\
\hline Lago Escondido ${ }^{1,3}$ & 1966 & N. pumilio & Raleos & Tierra del Fuego \\
\hline Río Milnak ${ }^{1,3}$ & 1966 & N. pumilio & Raleos & Tierra del Fuego \\
\hline Ea. Moat ${ }^{5}$ & 1993 & $N$. betuloides & Raleos & Tierra del Fuego \\
\hline Ea. San Justo ${ }^{6}$ & 1996 & N. pumilio & $\begin{array}{l}\text { Método de raleo para reducir } \\
\text { la caída por viento }\end{array}$ & Tierra del Fuego \\
\hline Ea. Stag River & 1996 & N. pumilio & $\begin{array}{l}\text { Raleos bajo diferentes niveles } \\
\text { de cobertura del dosel }\end{array}$ & Santa Cruz \\
\hline Ea. San Justo - Rodal $13^{7}$ & 1997 & N. pumilio & Cortas de regeneración & Tierra del Fuego \\
\hline Ea. San Justo - Rodal $4^{8}$ & 2001 & N. pumilio & $\begin{array}{l}\text { Cortas de regeneración con } \\
\text { retención variable }\end{array}$ & Tierra del Fuego \\
\hline Ea. Tres Marías ${ }^{10}$ & 2003 & N. antarctica & Sistemas silvopastoriles & Santa Cruz \\
\hline Ea. Cancha Carreras ${ }^{10}$ & 2003 & N. antarctica & Sistemas silvopastoriles & Santa Cruz \\
\hline Ea. Los Cerros ${ }^{9}$ & 2004 & N. pumilio & $\begin{array}{l}\text { Cortas de regeneración con } \\
\text { retención variable }\end{array}$ & Tierra del Fuego \\
\hline Ea. Nibepo Aike ${ }^{10}$ & 2004 & N. antarctica & Sistemas silvopastoriles & Santa Cruz \\
\hline
\end{tabular}

${ }^{1}$ Cozzo et al. (1967), ${ }^{2}$ Cozzo et al. (1969), ${ }^{3}$ Mutarelli \& Orfila (1969), ${ }^{4}$ Martínez-Pastur et al. (2001), ${ }^{5}$ MartínezPastur et al. (2002a), ${ }^{6}$ Peri et al. (2002), ${ }^{7}$ Martínez-Pastur et al. (1999), ${ }^{8}$ Martínez-Pastur et al. (2009), ${ }^{9}$ MartínezPastur et al. (2007), ${ }^{10}$ Peri et al. (2005). 
colaboración con empresas privadas. En particular, a mediados de la década del '90 se establecieron programas de colaboración para Patagonia sur entre instituciones nacionales (INTA y CONICET), universidades (Universidad Nacional de La Plata, Universidad Nacional de la Patagonia Austral y Universidad Nacional del Sur), establecimientos agropecuarios y empresas forestales (aserraderos y carpinterías industriales). Los objetivos primarios fueron mejorar la rentabilidad de las industrias, a partir del estudio de la biometría y el rendimiento durante el proceso de aprovechamiento (e.g., MartínezPastur et al. 2000) y definir la aplicabilidad del manejo forestal a largo plazo en Patagonia Sur. Para ello se estableció una red de parcelas permanentes para estudios a largo plazo. Las primeras parcelas se establecieron para determinar mejoras en las prácticas silvícolas de bosques secundarios de $N$. pumilio y $N$. betuloides (Tabla 1, Fig. 1), ensayando diferentes estrategias de manejo mediante la aplicación de raleos y podas: (i) Ea. Moat (desde 1993) de 1 ha, (ii) Ea. San Justo (desde 1996) en 2 ha, y (iii) Ea. Stag River (desde 1996) en 2 ha (Martínez-Pastur et al. 1997, 2001, 2002a, Peri et al. 2002). Luego se estableció una parcela permanente en la Ea. San Justo - Rodal 13 (desde 1997) en 2 ha para definir las estrategias de cosecha más efectivas y su impacto en la regeneración forestal (Martínez-Pastur et al. 1999). Mediante estas parcelas fue posible obtener datos valiosos

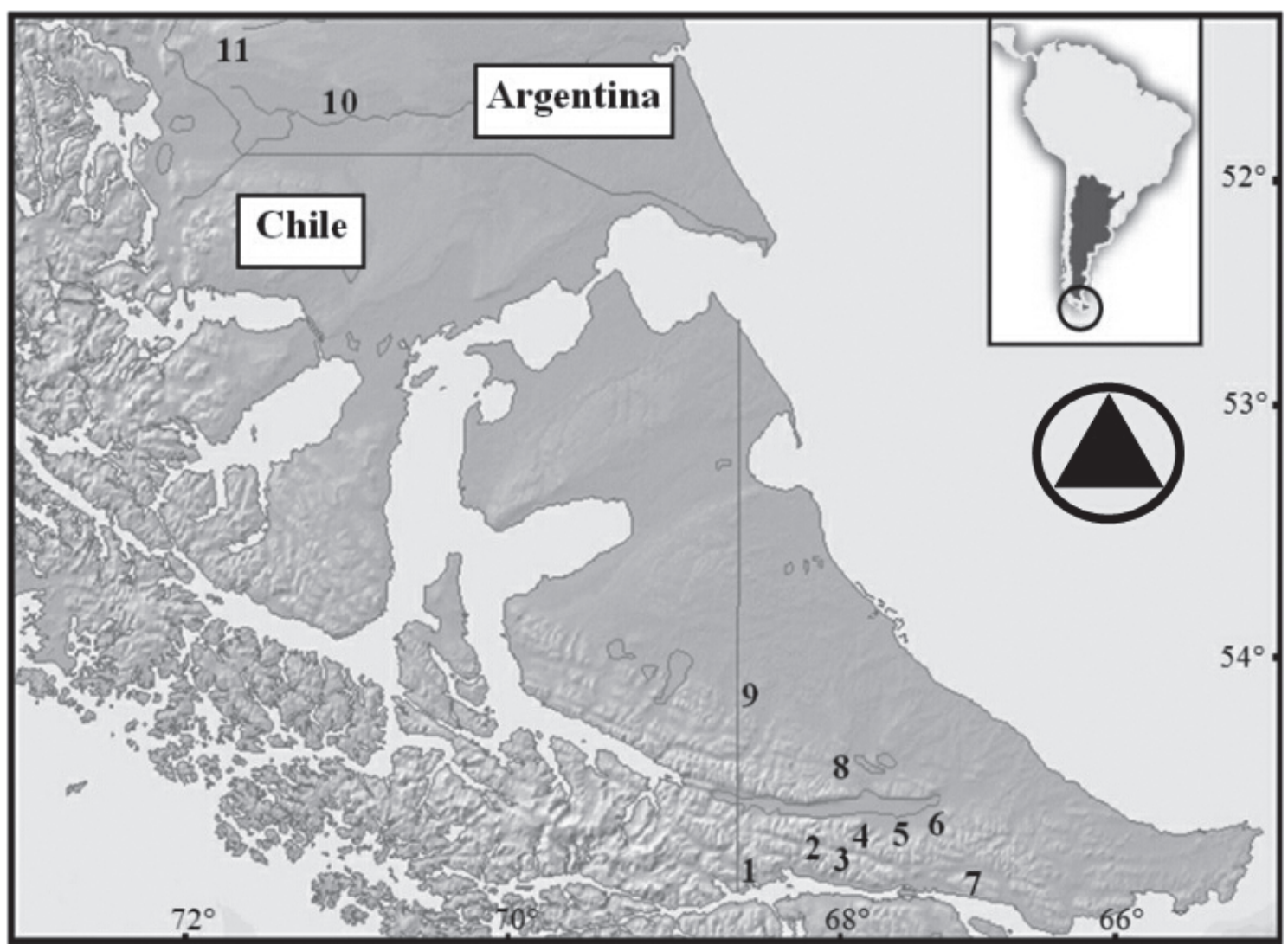

Fig. 1: Ubicación de las parcelas de investigación a largo plazo en el sector argentino de Patagonia Sur.

Location of long-term research sites in the Argentine portion of Southern Patagonia.

1 = Laguna Negra, Lago Roca, Cañadón del Toro, Lapataia y Ensenada; 2 = Río Tristen, Vega Café y Monte Redondo; 3 = Río Tierra Mayor; 4 = Lago Escondido; 5 = Río Milnak; 6 = Agua Blanca; 7 = Ea. Moat; 8 = Ea. Los Cerros; 9 = Ea. San Justo; 10 = Ea. Stag River; 11 = Ea. Tres Marías y Ea. Cancha Carreras. 
para analizar la aplicabilidad económica de diferentes tratamientos de raleos (e.g., Fig. 2), o la efectividad de una práctica silvícola, por ejemplo, una corta de protección utilizando el dosel remanente y la dinámica del banco de plántulas (establecimiento, crecimiento y mortalidad) como indicadores (Fig. 3).

Durante los últimos diez años, varias empresas forestales comenzaron a analizar la posibilidad de acceder a nuevos mercados internacionales, debiendo para ello primero certificar sus actividades (e.g., Forest Stewardship Council). En este marco se llevaron a cabo varios estudios en bosques de $N$. pumilio sobre impacto del manejo forestal en la biodiversidad del bosque (Deferrari et al. 2001, Spagarino et al. 2001, Martínez-Pastur et al. 2002b) y su compartimentalización a nivel de paisaje (Lencinas et al. 2007, 2008a, 2008b). Consecuentemente, se firmaron acuerdos a largo plazo para estudiar distintas alternativas de manejo forestal basados en la retención variable en una parcela en la Ea. San Justo Rodal 4 (desde 2001) en 61 ha de bosques (Martínez-Pastur et al. 2009), principalmente sobre variables de biodiversidad (Ducid et al. 2005, Lencinas et al. 2007, 2008c, 2009). Finalmente, se estableció una parcela la Ea. Los Cerros (desde 2004) en 70 ha de bosque nativo para analizar a largo plazo la implementación de sistemas de cosecha con retención variable en el marco de un plan de manejo de una empresa forestal mediana (Aserradero Kareken) y con el soporte económico de los proyectos PIARFON de la SAyDS y de la Agencia Nacional de Promoción Científica y Tecnológica de la Nación Argentina.

De igual manera, en los últimos años creció el interés para establecer propuestas de

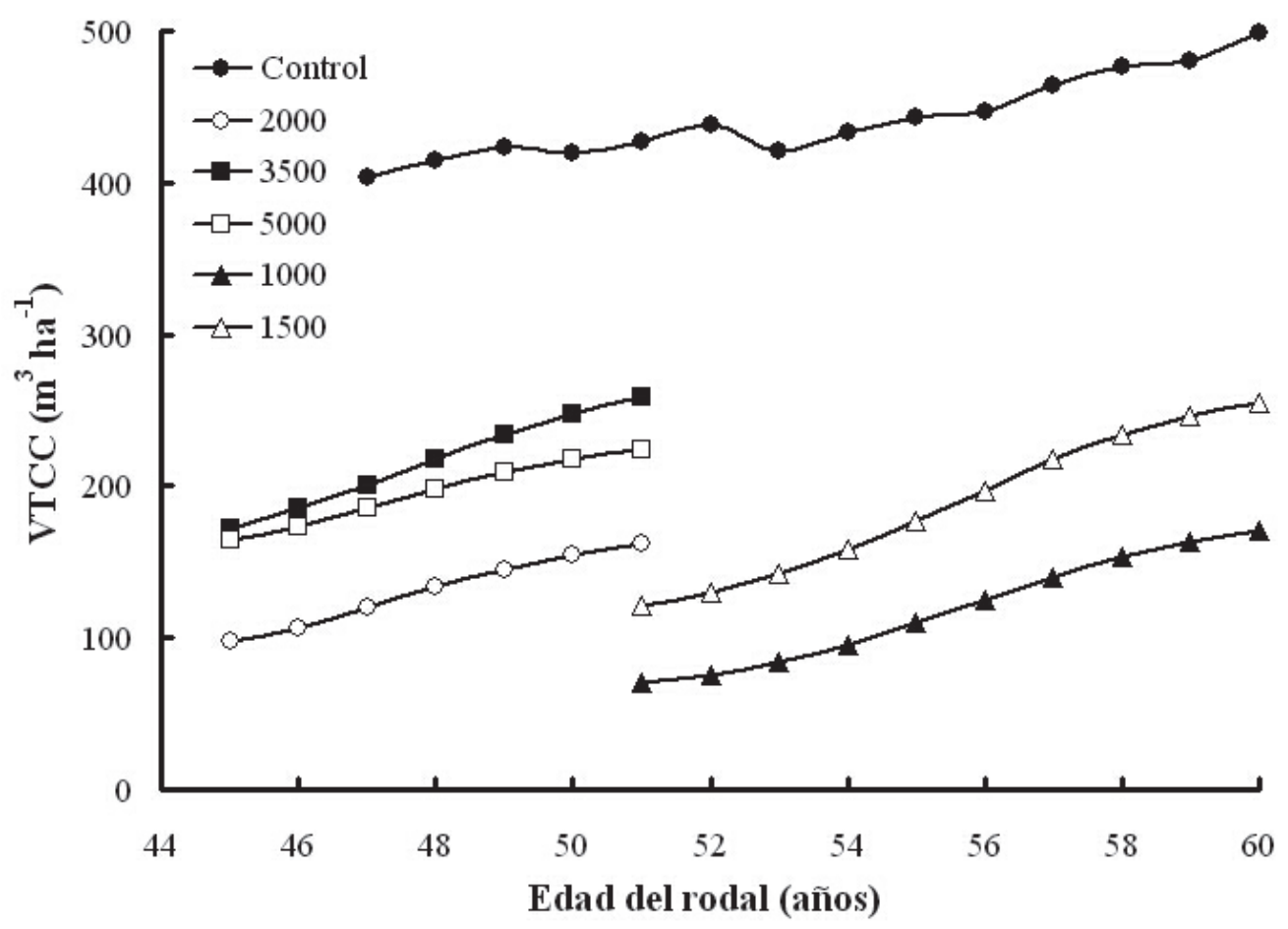

Fig. 2: Ejemplo de datos obtenidos en parcelas de estudio a largo plazo: volumen total con corteza (VTCC) de rodales raleados (1,000 a 5,000 árboles ha-1) y testigo en bosques de Nothofagus betuloides en Ea. Moat (Tierra del Fuego - Argentina) (los datos desde 45 a 51 años del rodal están publicados en Martínez-Pastur et al. 2002a y desde 51 a 59 años del rodal son datos inéditos).

Example of data obtained in long-term research plots: total over bark volume (VTCC) of thinned (1,000 to 5,000 trees $\mathrm{ha}^{-1}$ ) and control unthinned stands in Nothofagus betuloides forests from Moat Ranch plot (Tierra del Fuego Argentina) (data from 45 to 51 years of the stand were published in Martínez-Pastur et al. 2002a and from 51 to 59 years of the stand are unpublished data). 
manejo sustentable para los bosques de $N$. antarctica (Foster) Oerst. en Patagonia (Peri et al. 2005, Peri 2005, 2006). Para ello se establecieron tres parcelas de estudios a largo plazo para monitorear el manejo silvopastoril analizando diferentes intensidades de raleo, la mejora en la producción de los pastizales, la carga animal, y para proveer estrategias de preservación del estrato de árboles nativos en el tiempo: (i) Ea. Tres Marías (desde 2003) de 6 ha, (ii) Ea. Cancha Carreras (desde 2003) en 16 ha, y (iii) Ea. Nibepo Aike (desde 2004) en 5 ha, esta última en el marco de los proyectos PIARFON de la SAyDS (Peri et al. 2005).

La presente red de parcelas permanentes de estudio a largo plazo tuvo en cuenta las lecciones del pasado, tratando de mejorar la aplicabilidad de los planes de monitoreo en el futuro: (i) las nuevas parcelas se instalaron sobre terrenos privados, (ii) la ubicación, diseño y establecimiento de las parcelas se realizó en conjunto con las empresas privadas y establecimientos agropecuarios, (iii) se firmaron acuerdos entre instituciones nacionales para el estudio y seguimiento de los ensayos, (iv) se postularon a proyectos de corto plazo de financiamiento compartido entre instituciones nacionales y las empresas privadas participantes para el seguimiento $\mathrm{y}$ toma de datos en la red de parcelas instaladas, y (v) se establecieron políticas de concientización entre empresarios y dueños de establecimientos agropecuarios sobre la importancia de los estudios forestales a largo plazo. Entre las principales falencias encontradas en este proceso estuvieron: (i) la falta de compromiso del Gobierno (e.g., Dirección de Bosques de la Provincia), (ii) la ausencia de proyectos financiados a largo plazo (> 5 años), y (iii) la responsabilidad de la continuidad del estudio de las parcelas continúa bajo la exclusiva iniciativa de los investigadores científicos. Finalmente, es importante resaltar que muchos de los estudios en parcelas permanentes comparan los valores de las variables antes y después de las prácticas silvícolas (Before-After-ControlImpact o enfoque $\mathrm{BACI}$ ) para determinar la extensión de la variación en la biodiversidad antes de la implementación del tratamiento (Wardell-Johnson \& Williams 2000, Watherhouse \& Armleder 2007).

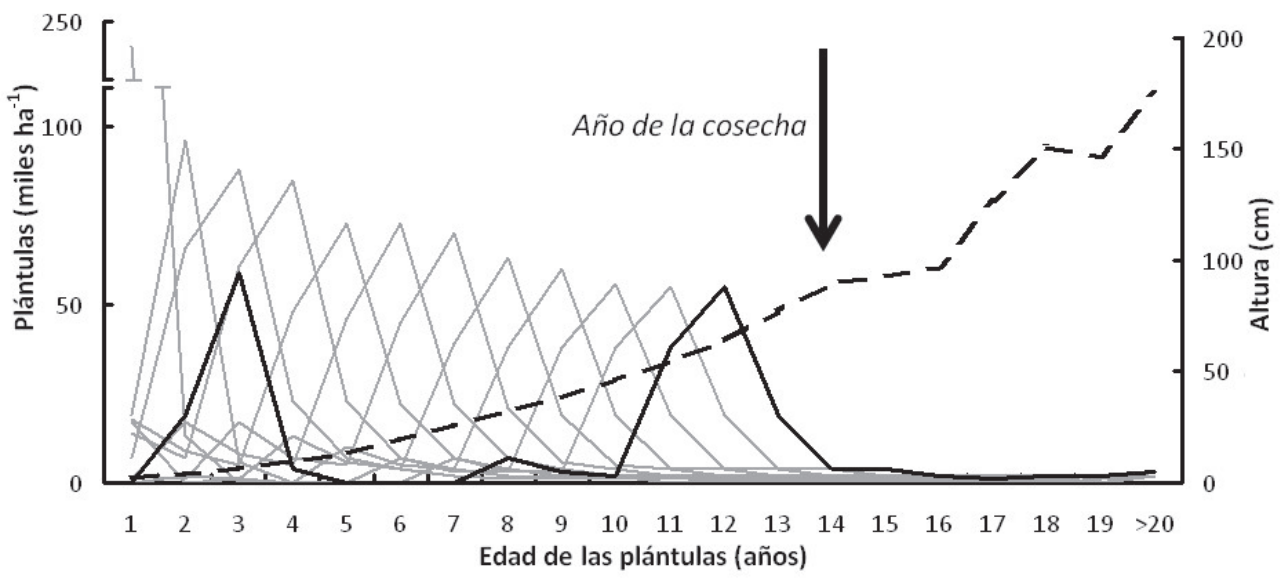

Fig. 3: Ejemplo de datos obtenidos en parcelas de estudio a largo plazo: estructura de edades del banco de plántulas en un bosque de Nothofagus pumilio cosechado hace 12 años en la Ea. San Justo Rodal 13 (Tierra del Fuego - Argentina). Línea negra = estructura de edades de la regeneración en el año 2009, líneas grises = estructura de edades de la regeneración en los años 2001-2008, línea punteada = altura de las plántulas. La flecha indica el año de la cosecha, donde las plántulas que aparecen a la derecha de la flecha se establecieron previo a la corta (datos inéditos).

Example of data obtained in long-term research plots: age structure of Nothofagus pumilio seedling bank in a harvested stand 13 years ago in San Justo Ranch - Stand 13 (Tierra del Fuego - Argentina). Black line = seedlings age structure in year 2009, grey lines = seedlings age structure in years 2001-2008, dotted line = seedling height. Arrow indicates the harvesting date, where seedlings density data at the right of the arrow were established prior to harvesting (unpublished data). 


\section{ESTUDIOS A LARGO PLAZO SOBRE MANEJO FORESTAL EN PATAGONIA SUR}

Los seguimientos en las parcelas permanentes de estudio incluyeron parámetros económicos, silvícolas y ecológicos (Tabla 2). Los parámetros económicos y silvícolas incluyeron: (i) estimaciones de crecimiento individual y de rodal en rodales intervenidos y no intervenidos (e.g., Ea. Moat) (Fig. 2) bajo diferentes condiciones de copa (e.g., Ea. Stag River), diferentes consideraciones económicas de implementación de raleos incluyendo la aplicación de podas (e.g., Agua Blanca), estrategias para reducir el riesgo de volteo de viento (e.g., Ea. San Justo), o la aplicación de sistemas silvopastoriles (e.g., Ea. Tres Marías,
Ea. Cancha Carreras, Ea. Nibepo Aike) (Martínez-Pastur et al. 2001, 2002a, Peri et al. 2002, 2005); (ii) rendimiento de cosecha con destino aserrado o leña en bosques secundarios (e.g., Ea. San Justo - Rodal 13), retención variable (e.g., Ea. San Justo - Rodal 4) o la aplicación de sistemas silvopastoriles (e.g., Ea. Tres Marías, Ea. Cancha Carreras, Ea. Nibepo Aike) (Martínez-Pastur et al. 1999, 2009, Peri et al. 2002, 2005); y (iii) dinámica del dosel remanente luego de las cortas (e.g., Ea. San Justo - Rodales 4 y 13, Ea Los Cerros, Ea. Nibepo Aike) (Peri et al. 2005, MartínezPastur et al. 2007, 2009).

Los parámetros ecológicos incluyeron: (i) patrones de producción de flores y semillas, asi como dinámica de la regeneración en cortas de

TABLA 2

Investigación llevada a cabo en las actuales parcelas de estudio a largo plazo en Patagonia Sur. Research studies carried out in the current long-term plots of Southern Patagonia.

\begin{tabular}{|c|c|c|c|c|c|c|c|c|c|c|}
\hline \multirow[b]{2}{*}{$\begin{array}{l}\text { Estudios de } \\
\text { investigación }\end{array}$} & \multicolumn{10}{|c|}{ Parcelas } \\
\hline & 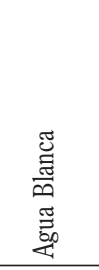 & 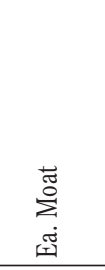 & 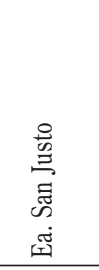 & 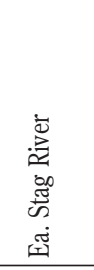 & 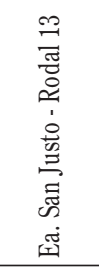 & 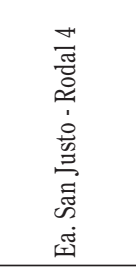 & 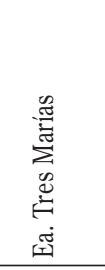 & 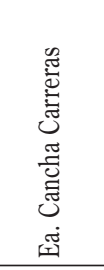 & 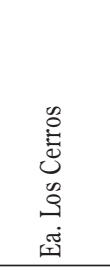 & 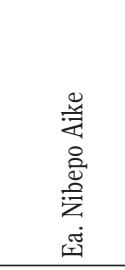 \\
\hline Tratamientos de raleo & $1985 \mathrm{P}^{1}$ & $1993 \mathrm{P}^{2}$ & $1996 \mathrm{P}^{3}$ & $1996 \mathrm{P}$ & & & $2003 \mathrm{P}^{9}$ & $2003 \mathrm{P}^{9}$ & & $2004 \mathrm{P}^{9}$ \\
\hline Tratamientos de poda & $1999 \mathrm{P}$ & & & & & & & & & \\
\hline \multicolumn{11}{|l|}{ Dinámica de rodal de } \\
\hline Bosques naturales & $1999 \mathrm{P}$ & $1993 \mathrm{P}^{2}$ & $1996 \mathrm{P}^{3}$ & $1996 \mathrm{P}$ & & & & & & $2004 \mathrm{P}^{9}$ \\
\hline Rendimiento forestal & & & $1999^{3}$ & & $1997^{4}$ & $2001^{5}$ & $2003^{9}$ & $2003^{9}$ & & $2004^{9}$ \\
\hline Dinámica del dosel remanente & & & & & $1997 \mathrm{P}$ & $2001 \mathrm{P}^{5}$ & & & $2005 \mathrm{P}^{7}$ & $2004 \mathrm{P}^{9}$ \\
\hline Producción de semillas & & & & & & $2002 \mathrm{P}$ & $2003 \mathrm{P}^{9}$ & $2003 \mathrm{P}^{9}$ & $2006 \mathrm{P}^{7,8}$ & $20042006^{9}$ \\
\hline Dinámica de la regeneración & & & & & $1997 \mathrm{P}^{4}$ & $2001 \mathrm{P}$ & $2003 \mathrm{P}^{9}$ & $2003 \mathrm{P}^{9}$ & $2004 \mathrm{P}^{7}$ & \\
\hline Variables microclimáticas & & & & & & 20022005 & $2003 \mathrm{P}^{9}$ & $2003 \mathrm{P}^{9}$ & $2005 \mathrm{P}^{7}$ & $20042006^{9}$ \\
\hline Biodiversidad & & & & & 1997 & $20012005^{6}$ & $2003 \mathrm{P}^{9}$ & $2003 \mathrm{P}^{9}$ & $2005 \mathrm{P}^{7}$ & $20042006^{9}$ \\
\hline Pasturas & & & & & & & $2003 \mathrm{P}^{9}$ & $2003 \mathrm{P}^{9}$ & & $20042006^{9}$ \\
\hline Estudios ecofisiológicos & & & & & & & 2007 & 2007 & $2005 \mathrm{P}$ & \\
\hline Producción de hojarasca & & & & & & $2002 \mathrm{P}$ & $2003 \mathrm{P}^{9}$ & $2003 \mathrm{P}^{9}$ & $2006 \mathrm{P}$ & \\
\hline Ciclos de nutrientes & & & & & & 20032007 & $2003 \mathrm{P}^{9}$ & $2003 \mathrm{P}^{9}$ & $2006 \mathrm{P}^{7}$ & \\
\hline Propiedades del suelo & & & & & & $2003 \mathrm{P}$ & $2003 \mathrm{P}$ & $2003 \mathrm{P}$ & $2006 \mathrm{P}^{7}$ & \\
\hline
\end{tabular}

$\mathrm{P}=$ hasta el presente (2008). Resultados parciales aparecen publicados en: ${ }^{1}$ Martínez-Pastur et al. (2001), 2 Martínez-Pastur et al. (2002a), ${ }^{3}$ Peri et al. (2002), ${ }^{4}$ Martínez-Pastur et al. (1999), ${ }^{5}$ Martínez-Pastur et al. (2009), 6 Ducid et al. (2005) and Lencinas et al. (2007, 2008c, 2009), ${ }^{7}$ Martínez-Pastur et al. (2007), ${ }^{8}$ Martínez-Pastur et al. (2008), ${ }^{9}$ Peri et al. (2005). 
protección, retención variable y sistemas silvopastoriles considerando gradientes de coberturas de copas (e.g., Ea. San Justo - Rodal 4 y 13, Ea. Los Cerros) (Fig. 3), y disponibilidad hídrica en el suelo (e.g., Ea. Tres Marías, Ea. Cancha Carreras, Ea. Nibepo Aike) (MartínezPastur et al. 1999, 2007, 2008, Peri et al. 2005); (ii) variables microclimáticas medidas mediante estaciones climáticas (Davis Weather Wizard III y accesorios - USA) y data loggers (HOBO ONSET y Watchdog Spectrum - USA) para analizar temperaturas del aire y del suelo, humedad relativa del aire, velocidad y dirección del viento, precipitaciones y humedad del suelo (Watermark Spectrum - USA) (Peri et al. 2005, Martínez-Pastur et al. 2007); (iii) biodiversidad, incluyendo aves, insectos, plantas superiores, helechos, musgos y hongos (Peri et al. 2005, Ducid et al. 2005, Lencinas et al. 2007, 2008c, 2009, Martínez-Pastur et al. 2007); (iv) productividad de pasturas para diferentes alternativas de sistemas silvopastoriles (e.g., Ea. Tres Marías, Ea. Cancha Carreras, Ea. Nibepo Aike) (Peri et al. 2005); (v) estudios ecofisiológicos, incluyendo fotosíntesis, estrés de humedad de las plantas, compartimentalización de la biomasa y requerimientos de luz; y (vi) producción de hojarasca, ciclos de nutrientes (descomposición, mineralización e inmovilización en $\mathrm{N}$ y $\mathrm{P}$ ) y cambios en las propiedades del suelo en tratamientos de retención variable y sistemas silvopastoriles (Peri et al. 2005, Martínez-Pastur et al. 2007). Además de esto, distintas variables bióticas y abióticas no fueron incluidas en el presente muestreo, pero deberían incluirse para mejorar la comprensión sobre los tratamientos analizados, e.g., hongos y líquenes, flora no vascular, ecología de especies afectadas (e.g., coleópteros), mamíferos incluyendo murciélagos, dinámica de la regeneración incluyendo competencia interespecífica 0 facilitación, insectos acuáticos, ciclo del agua, y aspectos sociales de las actividades económicas propuestas.

\section{COMENTARIOS FINALES}

La actividad forestal juega un importante papel en el desarrollo económico-social de Patagonia Sur, principalmente en Tierra del Fuego (Mutarelli \& Orfila 1969). Los distintos programas de investigación forestal en Argentina han demostrado la posibilidad de mejorar el uso sustentable de los bosques de Nothofagus, favoreciendo su regeneración, conservación y desarrollo, y por la estabilización de las industrias en defensa del patrimonio forestal nacional (Mutarelli \& Orfila 1973).

Las parcelas de ensayo a largo plazo han permitido: (i) proponer un nuevo sistema de cosecha para la maximización del rendimiento y mejorando el valor de conservación de los bosques bajo manejo (e.g., retención variable y sistemas silvopastoriles) (Martínez-Pastur et al. 2009, Peri 2005, 2006), (ii) determinar la factibilidad económica de la implementación de tratamientos intermedios, (iii) definir las líneas de base y los impactos de diferentes tratamientos silvícolas, (iv) proponer una metodología de monitoreo de amplio espectro y establecer áreas demostrativas de manejo forestal, y (v) proveer de áreas y conocimientos para entrenar profesionales en manejo forestal y prácticas de conservación de la biodiversidad. El inventario de la red de parcelas permanentes en bosques de Nothofagus de Patagonia Sur y la información obtenida durante los últimos 15 años podrían ser usadas como punto de partida para definir un núcleo regional que pudiera participar del International Long Term Ecological Research (ILTER) para mejorar el uso de los bosques nativos. Sin embargo, la red de investigación a largo plazo implementada no incluye las mismas metodologías de muestreo ni el mismo diseño o tamaño. Es importante resaltar la importancia de los futuros diseños de muestreo que puedan permitir obtener datos comparables con esta red y con otras redes alrededor del mundo.

La red actual de parcelas de investigación a largo plazo no es suficiente para definir las propuestas de manejo forestal sustentables para todos los tipos de bosque y en toda su variabilidad ambiental. Sin embargo, dichas parcelas pueden proveer de buenas herramientas de decisión con las cuales proponer nuevos lineamientos para un manejo adaptativo en un amplio espectro de objetivos (e.g., conservación de la biodiversidad y producción de madera para aserrado). Se espera que una mejor comprensión de los resultados obtenidos a partir de estudios a 
largo plazo, así como tener en cuenta la existencia de parcelas permanentes en Patagonia para futuras investigaciones, contribuirán a solucionar los desafíos ecológicos y socioeconómicos en el uso sustentable de nuestros bosques nativos. Sin embargo, las instituciones de investigación y administrativas, las compañías forestales, los estancieros y los aserraderos deben cooperar y adoptar un compromiso sostenido para financiar y mantener estas por demás únicas parcelas y plataformas de investigación a largo plazo.

\section{MATERIAL COMPLEMENTARIO}

La versión en inglés de este artículo está disponible como Material Complementario online en http://rchn.biologiachile.cl/ s u p p mat / $2010 / 1 / \mathrm{M} \mathrm{C}_{\text {_ }}$ Martin e $z$ Pastur_et_al_2010.pdf

\section{AGRADECIMIENTOS}

A los aserraderos y empresas que contribuyeron con la investigación a largo plazo en Patagonia Sur. A Ricardo Vukasovic (Servicios Forestales), Rosina Soler Esteban (CADIC), Horacio Ivancich (CADIC), Lucas Monelos (UNPA), Héctor Bahamonde (INTA) y Verónica Gargaglione (INTA) por su activa colaboración en los proyectos, y a los organizadores del taller internacional "Confronting global change with a network of long-term socio-ecological research sites in the south of the Americas" (MIDEPLAN ICM P05002, CONICYT PFB-23).

\section{LITERATURA CITADA}

ALFONSO J (1942) Los bosques de Tierra del Fuego. Revista Suelo Argentino (Argentina) 1: 47-51.

ALONSO O, E MUTARELLI \& E ORFILA (1968) Resultado de los tres primeros años del plan de investigaciones silviculturales y dasométricas necesarias para la organización económica de los bosques subantárticos argentinos. Revista Forestal Argentina (Argentina) 12: 3-31.

COSTANTINO I (1950) La lenga: Estudio forestal y método de tratamiento. Revista de la Facultad de Agronomía de La Plata (Argentina) 27: 197-220.

COZZO D, E MUTARELLI \& E ORFILA (1967) Plan de Investigaciones silviculturales y dasonómicas necesarias para la organización económica de los bosques subantárticos argentinos. Plan $\mathrm{n}^{\circ}$ 118. Convenio Cátedra de Dasonomía - UBA y CAFPTA.

COZZO D, E MUTARELLI \& E ORFILA (1969) Plan de Investigaciones silvo-dasocráticas en las etapas de ordenación, recuperación y reproducción económica de los bosques Andino-Patagónicos. Plan $\mathrm{n}^{\circ}$ 129. Convenio Cátedra de Dasonomía UBA y CAFPTA.

DEFERRARI G, C CAMILION, G MARTÍNEZ-PASTUR \& P PERI (2001) Changes in Nothofagus pumilio forest biodiversity during the forest management cycle: Birds. Biodiversity and Conservation 10: 2093-2108.

DUCID G, M MURACE \& JM CELLINI (2005) Diversidad fúngica en el filoplano de Osmorhiza spp. relacionado con el sistema de regeneración empleado en bosques de Nothofagus pumilio en Tierra del Fuego, Argentina. Bosque 26: 33-42.

FRANKLIN J, D BERG, D THORNBURGH \& J TAPPEINER (1997) Alternative silvicultural approaches to timber harvesting: Variable retention harvest systems. En: Kohm K \& J Franklin (eds) Creating a forestry for the $21^{\text {st }}$ Century: 111-140. Island press, New York, USA.

GEA G, G MARTÍNEZ-PASTUR, JM CELLINI \& MV LENCINAS (2004) Forty years of silvicultural management in southern Nothofagus pumilio (Poepp. et Endl.) Krasser primary forests. Forest Ecology and Management 201: 335-347.

GONZÁLEZ M, C DONOSO-ZEGERS, P OVALLE \& G MARTÍNEZ-PASTUR (2006) Nothofagus pumilio (Poepp. et Endl) Krasser - lenga, roble blanco, leñar, roble de Tierra del Fuego - Familia: Fagaceae. En: Donoso-Zegers C (ed) Las especies arbóreas de los bosques templados de Chile y Argentina: Autoecología: 486-500. Marisa Cúneo Ed., Valdivia, Chile.

LENCINAS MV, G MARTÍNEZ-PASTUR, M MEDINA \& C BUSSO (2005) Richness and density of birds in timber Nothofagus pumilio forests and their unproductive associated environments. Biodiversity and Conservation 14: 2299-2320.

LENCINAS MV, G MARTÍNEZ-PASTUR, E GALLO, A MORETTO, C BUSSO \& P PERI (2007) Mitigation of biodiversity loss in Nothofagus pumilio managed forests of South Patagonia. En: Pacha MJ, S Luque, L Galetto \& L Iverson (eds) Understanding biodiversity loss: An overview of forest fragmentation in South America. Part III: Landscape ecology for conservation, management and restoration: 112-120. IALE Landscape Research and Management papers.

LENCINAS MV, G MARTÍNEZ-PASTUR, C ANDERSON \& C BUSSO (2008a) The value of timber quality forests for insect conservation on Tierra del Fuego Island compared to associated non-timber quality stands. Journal of Insect Conservation 12: 461-475.

LENCINAS MV, G MARTÍNEZ-PASTUR, P RIVERO \& C BUSSO (2008b) Conservation value of timber quality vs. associated non-timber quality stands for understory diversity in Nothofagus forests. Biodiversity and Conservation 17: 2579-2597.

LENCINAS MV, G MARTÍNEZ-PASTUR, R SOLÁN, E GALLO \& JM CELLINI (2008c) Forest management with variable retention impact over moss communities of Nothofagus pumilio understory. Forstarchiv 79: 77-82. 
LENCINAS MV, G MARTÍNEZ-PASTUR, E GALLO \& JM CELLINI (2009) Alternative silviculture with variable retention to improve bird conservation in timber managed South Patagonia forests. Forest Ecology and Management 258: 472-480.

MARTÍNEZ-PASTUR G, P PERI, R VUKASOVIC, S VACCARO \& V PIRIZ-CARRILLO (1997) Site index equation for Nothofagus pumilio Patagonian forest. Phyton 6: 55-60.

MARTÍNEZ-PASTUR G, P PERI, MC FERNÁNDEZ \& G STAFFIERI (1999) Desarrollo de la regeneración a lo largo del ciclo del manejo forestal de un bosque de Nothofagus pumilio: 1 . Incidencia de la cobertura y el aprovechamiento o cosecha. Bosque 20: 39-46.

MARTÍNEZ-PASTUR G, JM CELLINI, P PERI, R VUKASOVIC \& C FERNÁNDEZ (2000) Timber production of Nothofagus pumilio forests by a shelterwood system in Tierra del Fuego (Argentina). Forest Ecology and Management 134: 153-162.

MARTÍNEZ-PASTUR G, JM CELLINI, MV LENCINAS, R VUKASOVIC, R VICENTE, F BERTOLAMI \& J GIUNCHI (2001) Modificación del crecimiento y de la calidad de fustes en un raleo fuerte de un rodal en fase de crecimiento óptimo inicial de Nothofagus pumilio (Poepp. et Endl.) Krasser. Ecología Austral (Argentina) 11: 95-104.

MARTÍNEZ-PASTUR G, JM CELLINI, MV LENCINAS, R VUKASOVIC, P PERI \& S DONOSO (2002a) Response of Nothofagus betuloides (Mirb.) Oersted to different thinning intensities in Tierra del Fuego (Argentina). Interciencia 27: 679-685.

MARTÍNEZ-PASTUR G, P PERI, MC FERNÁNDEZ, G STAFFIERI \& MV LENCINAS (2002b) Changes in understory species diversity during the Nothofagus pumilio forest management cycle. Forest Research 7: 165-174

MARTÍNEZ-PASTUR G \& MV LENCINAS (2005) El manejo forestal en los bosques de Nothofagus pumilio en Tierra del Fuego. IDIA XXI (Argentina) 5: 107-110.

MARTINEZ-PASTUR G, MV LENCINAS, P PERI, A MORETTO, JM CELLINI, I MORMENEO \& R VUKASOVIC (2007) Harvesting adaptation to biodiversity conservation in sawmill industry: Technology innovation and monitoring program. Technology Management and Innovation 2: 58-70.

MARTÍNEZ-PASTUR G, MV LENCINAS, P PERI \& JM CELLINI (2008) Flowering and seeding patterns in unmanaged and managed Nothofagus pumilio forests with a silvicultural variable retention system. Forstarchiv 79: 60-65.

MARTÍNEZ-PASTUR G, JM CELLINI, P PERI, MV LENCINAS, E GALLO \& R SOLER-ESTEBAN (2009) Timber management with variable retention in Nothofagus pumilio forests of Southern Patagonia. Forest Ecology and Management 258: 436-443.
MUTARELLI E \& E ORFILA (1969) Los bosques de Tierra del Fuego y los primeros ensayos de tratamientos para su regeneración, conducción y organización. Revista Forestal Argentina (Argentina) 13: 125-137.

MUTARELLI E \& E ORFILA (1971) Observaciones sobre la regeneración de lenga, Nothofagus pumilio (Poepp. et Endl.) Oerst., en parcelas experimentales del lago Mascardi, Argentina. Revista Forestal Argentina (Argentina) 15: 109115.

MUTARELLI E \& E ORFILA (1973) Algunos resultados de las investigaciones de manejo silvicultural que se realizan en los bosques AndinoPatagónicos de Argentina. Revista Forestal Argentina (Argentina) 17: 69-75.

PERI P, G MARTÍNEZ-PASTUR, R VUKASOVIC, B DÍAZ, MV LENCINAS \& JM CELLINI (2002) Thinning schedules to reduce risk of windthrow in Nothofagus pumilio forests of Patagonia, Argentina. Bosque 23: 19-28.

PERI P, G MARTÍNEZ-PASTUR, L MONELOS, M ALLOGIA, E LIVRAGHI, R CHRISTIANSEN \& MV STURZENBAUM (2005) Sistemas silvopastoriles en bosques nativos de ñire: Una estrategia para el desarrollo sustentable en la Patagonia Sur. En: Zárate R \& L Artesi (eds) Dinámicas Mundiales, Integración Regional y Patrimonio en Espacios Periféricos: 251-259. Universidad Nacional de la Patagonia Austral, Río Gallegos, Argentina.

PERI P (2005) Sistemas silvopastoriles en ñirantales. IDIA XXI (Argentina) 8: 255-259.

PERI P (2006) Sistemas silvopastoriles en bosques nativos de ñire de Patagonia Sur. SAGPyA Forestal (Argentina) 38: 1-7.

PITERBARG R (1965) Inventario y ordenación de un bosque de lenga (Nothofagus pumilio) en Tierra del Fuego, y primeros resultados de su explotación. Revista Forestal Argentina (Argentina) 9: 105-113.

ROTHKUGEL M (1916) Los bosques Andino Patagónicos. Ministerio de Agricultura. Dirección General de Agricultura y Defensa Agrícola. Buenos Aires, Argentina.

SPAGARINO C, G MARTÍNEZ-PASTUR \& P PERI (2001) Changes in Nothofagus pumilio forest biodiversity during the forest management cycle: Insects. Biodiversity and Conservation 10: 2077-2092.

WARDELL-JOHNSON G \& M WILLIAMS (2000) Edges and gaps in mature karri forest, southwestern Australia: Logging effects on bird species abundance and diversity. Forest Ecology and Management 131: 1-21.

WATHERHOUSE M \& H ARMLEDER (2007) Forest bird response to partial cutting in lodgepole pine forests on caribou winter range in west-central British Columbia. B.C. J. Ecosystems Management 8: 75-90. 
